\title{
Recomendaciones nutricionales en cuidados paliativos para pacientes con enfermedades avanzadas y al final de la vida
}

\author{
Nutritional recommendations in palliative care for patients with advanced \\ diseases and at the end of life \\ Recomendações nutricionais em cuidados paliativos para pacientes com doenças \\ avançadas e no final da vida
}

\author{
Giovanna Muscogiuri1 ${ }^{1}{ }^{*}$, Luigi Barrea ${ }^{1,2}$, María de los Ángeles Carignano ${ }^{3}$, Florencia Ceriani ${ }^{4}$
}

Recibido: 18 de enero de 2021. Aceptado para publicación: 25 de enero de 2021. https://doi.org/10.35454/rncm.v4n2.272

\begin{abstract}
Resumen
Los cuidados paliativos (CP) se basan en un abordaje multidisciplinar del paciente y su familia para prevenir y aliviar el sufrimiento del paciente, al promover y mantener una óptima calidad de vida hasta la muerte. La alimentación desempeña un papel fundamental en la vida de las personas. Más allá de la nutrición, el hecho de que las personas moribundas tengan anorexia como parte de sus síntomas es difícil de aceptar, ya que significa admitir la muerte. Un plan multidisciplinar de nutrición es fundamental, que se adapte al estadio de la enfermedad, siempre considerando que los beneficios deben superar los posibles efectos adversos. Como parte integral de la $\mathrm{CP}$, la nutrición e hidratación artificiales (NHA) pueden aumentar la supervivencia y mejorar la calidad de vida en pacientes seleccionados en función de su patología. Por tanto, el soporte nutricional debe prescribirse de acuerdo con el estadio de la enfermedad del paciente y los requerimientos energéticos y proteicos, analizando la tolerabilidad y, sobre todo, respetando los deseos del paciente, la familia y el cuidador. Es fundamental conocer sus preferencias y lograr una comunicación eficiente dentro del equipo de salud. Al hacerlo, es posible alcanzar un entendimiento compartido de los objeti-
\end{abstract}

\section{Summary}

Palliative care $(P C)$ is based on a multidisciplinary approach to the patient and their family to prevent and alleviate the suffering of the patient, promoting and maintaining an optimal quality of life until death. Food plays a fundamental role in people's lives. Beyond nutrition, the fact that dying people have anorexia as part of their symptoms is difficult to accept as it means admitting death. A multidisciplinary plan to nutrition is essential, adapting to the disease stage and always considering that the benefits must outweigh the possible adverse effects. As an integral part of $\mathrm{PC}$, artificial nutrition and hydration (ANH) can increase survival and improve quality of life in selected patients based on their pathology. Therefore, nutritional support should be prescribed according to the patient's disease stage, energy and protein requirements, analyzing tolerability, and above all, respecting the wishes of the patient, family or caregiver. It is essential to know the patient, family, or caregivers' preferences and achieve efficient communication within the health team. By doing so, it is possible to reach a shared understanding of the goals of palliative treatment among all members involved. This narrative review aims to describe the general and specific nutritional

\section{Resumo}

Os cuidados paliativos (CP) são baseados em uma abordagem multidisciplinar para o paciente e sua família para prevenir e aliviar o sofrimento do paciente, promovendo e mantendo uma ótima qualidade de vida até a morte. A alimentação desempenha um papel fundamental na vida das pessoas. Além da nutrição, o fato de que as pessoas moribundas apresentarem anorexia como parte dos sintomas é difícil aceitar, pois significa admitir a morte. Um plano multidisciplinar de nutrição é fundamental, adaptando-se ao estágio da doença e sempre considerando que os benefícios devem superar os possíveis efeitos adversos. Como parte integrante do $\mathrm{CP}$, a nutrição artificial e a hidratação (ANH) pode aumentar a sobrevida e meIhorar a qualidade de vida em pacientes selecionados com base em sua patologia. Portanto, o suporte nutricional deve ser prescrito de acordo com o estágio da doença do paciente, as necessidades energéticas e proteicas, analisando a tolerabilidade e, acima de tudo, respeitando os desejos do paciente/família/cuidador. É imprescindível conhecer as preferências do paciente/família/cuidadores e conseguir uma comunicação eficiente dentro da equipe de saúde. Com isso, é possível chegar a um entendimento compartilha- 
vos del tratamiento paliativo entre todos los miembros involucrados. Esta revisión narrativa tiene como objetivo describir las recomendaciones nutricionales generales y específicas en la etapa avanzada y al final de la vida de algunas de las enfermedades primarias en las que los $C P$ desempeñan un papel vital para ofrecer un tratamiento completo a estos pacientes específicos.

Palabras clave: cuidados paliativos, soporte nutricional, calidad de vida, final de la vida. recommendations in the advanced stage and at the end of life phase of some of the primary diseases in which PCs play a vital part in offering a complete treatment to these specific patients.

Keywords: Palliative Care; Nutritional Support; Quality of Life; End of Life. do dos objetivos do tratamento paliativo entre todos os membros envolvidos. Esta revisão narrativa tem como objetivo descrever as recomendações nutricionais gerais e específicas no estágio avançado e na fase final da vida de algumas das doenças primárias em que os CPs desempenham um papel vital na oferta de um tratamento completo a esses pacientes específicos.

Palavras-chave: cuidados paliativos, suporte nutricional, qualidade de vida, final de vida.
Departamento de Medicina Clínica y Cirugía, Unidad de Endocrinología, Federico II, University Medical School of Naples, Nápoles, Italia.

*Correspondencia: Giovanna Muscogiuri.

giovanna.muscogiuri@gmail.com
Centro Italiano de la Cura y el Bienestar del Paciente con Obesidad (CIBO),
departamento de Medicina Clínica y Cirugía, Unidad de Endocrinología,
Federico II University Medical School of Naples, Nápoles, Italia.
3 Unidad de Cuidad Intensivo, Sanatorio Franchín, Ciudad Autónoma de
Buenos Aires, Argentina.
4 Escuela de Nutrición, Universidad de la República, Montevideo, Uruguay.

manteniendo los aspectos éticos en el centro de los cuidados $^{(4)}$. Por tanto, esta revisión narrativa pretende describir las consideraciones nutricionales, generales y específicas, en la fase avanzada de algunas de las enfermedades primarias en las que los $\mathrm{CP}$ tiene un papel crucial, para ofrecer un tratamiento completo a este grupo particular de pacientes. Se resumirá la información actualizada buscada en PubMed y Google Scholar.

\section{CONSIDERACIONES NUTRICIONALES EN LOS CP}

La alimentación tiene profundas implicaciones en nuestra vida cotidiana, que van más allá de la propia nutrición. Comer implica una socialización y tiene profundas raíces culturales. Alimentar a los bebés, a los niños y a los ancianos y enfermos es algo instintivo, ya que se trata de supervivencia, lo cual está ampliamente aceptado. El hecho de que las personas muy enfermas y moribundas tengan anorexia como parte de sus síntomas y tiendan a no comer o a "no comer lo suficiente" es mucho más difícil de aceptar, porque significa aceptar la muerte.

Alimentar o no a las personas al final de la vida (FdV) es un tema muy complejo, el cual debe abordarse con una comunicación exhaustiva entre el paciente, la familia, los cuidadores y el equipo sanitario que lo atiende. Cuando la alimentación ya no contribuye a mejorar la enfermedad o la vida, se considera inútil presionar a los pacientes moribundos para que ingieran alimentos ${ }^{(5)}$. 
Esta última afirmación tiene más repercusión cuando el paciente no puede ingerir alimentos por vía oral, entonces la nutrición e hidratación artificial (NHA) se convierte en la única forma de ofrecer nutrientes a este grupo de pacientes; por ejemplo, los que se encuentran en fase terminal de demencia, o cuando la nutrición oral o la utilización de la sonda digestiva está contraindicada (cánceres gástricos, afectación peritoneal de cánceres abdominales avanzados) ${ }^{(6)}$.

La alimentación artificial es una práctica médica que debe ser prescrita, y necesita una sólida base científica para su aplicación; además, sus beneficios deben ser tangibles y superar sus posibles riesgos ${ }^{(7)}$. De lo contrario, la NHA es fútil y los principios éticos deben estar siempre presentes a la hora de tomar decisiones sobre cualquier tratamiento médico, incluidas las intervenciones nutricionales.

En este sentido se recomienda realizar un abordaje multidisciplinario de la desnutrición en los pacientes con enfermedades potencialmente mortales, para permitir un plan escalonado, que satisfaga las necesidades nutricionales específicas y dirigidas en las diferentes etapas de cada enfermedad. Este tipo de método puede identificar la desnutrición de forma temprana o incluso prevenir los trastornos nutricionales. Permite una intervención nutricional adecuada mediante la mejora de la nutrición oral o artificial, como la nutrición enteral (NE), a través de sonda nasogástrica (SNG), gastrostomía endoscópica percutánea (PEG) o nutrición parenteral (NP). Además, puede mejorar la CdV de los pacientes que reciben tratamientos específicos, como la terapia dirigida al tumor. Más adelante, en enfermedades avanzadas o terminales, la alimentación de confort puede formar parte de los CP, o incluso del cese de la terapia nutricional ${ }^{(6)}$.

\section{NUTRICIÓN PALIATIVA}

Hay varias consideraciones para tener en cuenta a la hora de diseñar estrategias nutricionales en CP. Como ya se ha dicho, el objetivo principal de los CP es fomentar y mantener la mejor CdV posible desde el momento en que se diagnostican las enfermedades potencialmente mortales. Como parte integral de los $\mathrm{CP}$, la NHA puede aumentar la supervivencia y mejorar la $\mathrm{CdV}$ en pacientes seleccionados con enfermedades neurológicas y cáncer, entre otras ${ }^{(7)}$. No obstante, cada enfermedad que pueda necesitar CP debe abordarse por separado. Además, es necesario diferenciar a los pacientes moribundos o en fase terminal de una enfer- medad en la que es más importante aliviar los síntomas que prolongar la supervivencia del paciente ${ }^{(8)}$.

\section{COMPLICACIONES DEL APOYO NUTRICIONAL}

Cuando se diseña un enfoque nutricional para cualquier paciente, como en toda intervención terapéutica, el primer paso es justificar la terapia; luego, los beneficios deben superar los posibles efectos adversos. Hay que tener en cuenta la enfermedad primaria, otros tratamientos adyuvantes/coadyuvantes, la medicación, las comorbilidades y el pronóstico. Una vez implantado el tratamiento decidido, hay que realizar un estrecho seguimiento para conseguir la eficacia y eficiencia establecidas como objetivo, y prevenir y tratar las complicaciones que puedan surgir.

Se ha demostrado ampliamente que las terapias nutricionales guiadas y controladas por equipos especializados y multidisciplinares alcanzan mejores resul$\operatorname{tados}^{(9-12)}$. En general, y teniendo en cuenta que la fisiopatología y los tratamientos están fuera del alcance de este trabajo, las complicaciones del soporte nutricional se pueden clasificar en:

- Secundarias a la implantación del acceso nutricional: SNG, PEG, acceso vascular para NP.

- Por desequilibrios metabólicos e hídricos.

- Complicaciones infecciosas.

En el caso de los pacientes en CP, mayormente aquellos con demencia o pacientes de edad avanzada, la NHA debe ser vigilada muy de cerca debido a sus múltiples comorbilidades. Esta acción implica consultas nutricionales y pruebas de laboratorio. Por supuesto, la frecuencia depende del tipo de terapia nutricional: más frecuente en la NP, menos en la NE y la suplementación ${ }^{(13)}$. Esta variación se debe a la sobrecarga de líquidos y a los desequilibrios metabólicos, que son muy frecuentes y pueden provocar disnea, complicaciones cardiovasculares y renales. Las complicaciones gastrointestinales, como la diarrea y el estreñimiento, son frecuentes en estas poblaciones. La NE también puede interferir en la absorción de la medicación, lo que puede dar lugar a complicaciones más graves.

Otro aspecto por analizar es la permanencia del acceso nutricional. Los pacientes con demencia y de edad avanzada tienden a referir molestias con este acceso $y$, en algunos casos, pueden desalojarlos, bloquearlos o $\operatorname{arrancarlos}^{(14)}$. La alimentación por sonda puede provocar úlceras por presión y aspiración recurrente. Esto 
puede provocar lesiones en los tejidos blandos, hospitalización por pérdida de sangre y necesidad de reinserción del acceso o infecciones. Para evitar estas complicaciones se suele sujetar a los pacientes, lo que se considera, claramente, que aumenta el sufrimiento de estos, y contrarresta los posibles beneficios que los médicos o las familias esperan de este tipo de tratamiento.

\section{APROXIMACIÓN A LOS CONCEPTOS DE LA ÉTICA BIOMÉDICA}

$\mathrm{Al}$ referirnos a la ética hay que tener en cuenta los principios relativos a la conducta correcta para lograr una práctica ética elevada en el soporte nutricional en el FdV, como en cualquier otra práctica médica. Estos principios son la beneficencia, la no maleficencia, el respeto a la autonomía y la justicia. Algunos autores conciben que estos principios solo pueden alcanzarse cuando los CP están centrados en el paciente, y se facilita la comunicación entre los miembros implicados ${ }^{(4)}$. Sin embargo, la decisión de iniciar, retener o retirar el soporte nutricional en el contexto del tratamiento paliativo sigue siendo un reto.

Una de las prioridades es conocer los deseos del paciente, la familia o los sustitutos en relación con las terapias de mantenimiento de la vida; en estos aspectos, la obtención de directivas avanzadas es obligatoria. Sin embargo, la mayoría de las instituciones médicas y los centros de salud no cuentan con los protocolos necesarios para comprometerse efectivamente con los pacientes y las familias para tener un plan, de acuerdo con sus deseos y creencias, para la atención anticipada ${ }^{(1)}$. Estos procesos requieren un cambio cultural entre los profesionales de la salud y el propio sistema sanitario, especialmente cuando la toma de decisiones se realiza al $\mathrm{FdV}^{(2)}$.

Existe evidencia en la literatura sobre la eficacia de la intervención clínica y nutricional para mejorar los resultados y la CdV de los CP. Hay un vacío a la hora de trasladar estos resultados e intervenciones probadas a la práctica clínica. La comunicación efectiva parece ser la clave para reducir esta brecha; esto significa conocer los deseos del paciente/familia y de los cuidadores, por un lado, y la comunicación temprana de los objetivos de la $\mathrm{CP}$ y dentro de un equipo multidisciplinar por parte del sistema sanitario.

\section{PERCEPCIÓN DE LA FAMILIA Y LOS CUIDADORES}

La anorexia y la imposibilidad de tomar alimentos por vía oral, síntomas comunes presentes en las enfermeda- des terminales y el cáncer, se encuentran entre las principales causas de angustia no solo para el paciente, sino para la familia y los cuidadores ${ }^{(15)}$. Además, los diferentes puntos de vista sobre la necesidad y los objetivos del tratamiento nutricional entre el equipo sanitario $y$ el paciente/familia pueden desencadenar un conflicto, que dificulta el proceso de toma de decisiones respecto a la práctica clínica.

Sin duda, los problemas de comunicación y las consecuencias emocionales están en el punto de mira. La formación y la educación en materia de comunicación son necesarias para lograr una comprensión compartida de los objetivos de los tratamientos paliativos entre todos los miembros implicados. Sin embargo, puede haber conflicto si no se igualan las expectativas de los distintos miembros comprometidos en el proceso, lo que es muy frecuente a la hora de decidir el abordaje nutricional y de hidratación en un entorno paliativo, debido al componente cultural de la nutrición ${ }^{(16)}$.

Por otro lado, la atención al malestar psicosocial que sufren los familiares de los pacientes con deterioro nutricional, las alteraciones de la familia y la relación del equipo sanitario son prioridades relacionadas con el logro de la mejor CdV posible ${ }^{(17)}$. En la revisión de Amano y colaboradores, los autores sugirieron una atención integrada paliativa, de cuidado nutricional, actuando de forma interdisciplinar, con acciones dirigidas a prevenir o tratar el malestar relacionado con la alimentación que los pacientes y familiares desarrollan en el contexto de los $\mathrm{CP}^{(17)}$.

Este enfoque tiene como objetivo reducir el conflicto y lograr la mejor CdV posible. En un estudio cualitativo sobre las experiencias del personal y de los familiares en relación con la CP, Francois y colaboradores sugirieron la aplicación de reuniones familiares para evitar/prevenir y desescalar el conflicto, y de auditorías clínicas para identificar y desarrollar estrategias para apoyar emocionalmente a los miembros de la familia. La técnica de resolución de conflictos ha tenido un impacto positivo en los resultados del tratamiento, en la CdV esperada e incluso en el proceso de duelo ${ }^{(16)}$.

\section{TRASTORNOS DE LA DEGLUCIÓN Y CP}

La disfagia y los trastornos de la deglución son frecuentes, especialmente entre las personas mayores y los pacientes debilitados. Estos pueden presentarse como un síntoma o una complicación en una amplia gama de enfermedades, tanto en el hogar como en el ámbito hospitalario. Su diagnóstico precoz es de cre- 
ciente interés por las complicaciones que puede ocasionar (aspiración, neumonía, deterioro nutricional), y la carga que representa para el equipo de tratamiento, el propio paciente y los cuidadores ${ }^{(18)}$. La complejidad de su reconocimiento y tratamiento exige, en la mayoría de los casos, un equipo multidisciplinar, en el que los logopedas o terapeutas de lenguaje desempeñan un papel importante.

A la hora de abordar la disfagia y su tratamiento es obligatorio diferenciar entre los pacientes con disfagia intratable, a pesar de todos los intentos, y el paciente moribundo con trastornos de la deglución. Esto se debe a que el objetivo para el primer grupo de pacientes es maximizar la función de la deglución, y mantener el estado nutricional y la salud pulmonar. En este sentido, el fracaso puede conducir a una PEG para la mejora nutricional y a opciones quirúrgicas invasivas ${ }^{(19)}$.

El segundo grupo de pacientes con enfermedades terminales que amenazan la vida necesita un enfoque de CP, como se ha dicho antes. En estos casos, la decisión de recurrir a terapia nutricional artificial debe tomarse con mucho cuidado, teniendo en cuenta el pronóstico y la $\mathrm{CdV}$ esperada. Los pacientes, la familia y los cuidadores deben participar en la decisión. En este sentido, toda persona incluida en la preparación de las comidas y la alimentación de estos pacientes tiende a responder al fracaso del tratamiento. Se ha demostrado que existe una relación entre la $\mathrm{CdV}$ del cuidador y la del paciente, sobre todo porque vivir y cuidar a una persona con disfagia implica responder a las necesidades físicas específicas del trastorno, pero también repercute en las actividades sociales, lo que modifica su estilo de vida y provoca una peor $\mathrm{CdV}$ según la escala de necesidades ${ }^{(20)}$.

El estudio cualitativo de DysCord, uno de los pocos realizados sobre el tema, mostró que los cuidadores tienden a presentar un fuerte deseo de continuar con la alimentación, y que los principales problemas encontrados fueron el conocimiento, la respuesta emocional y el papel simbólico de la comida. Cualquier discordancia con la recomendación dietética debe ser abordada, proporcionándoles herramientas para entender las implicaciones y el apoyo emocional para afrontarlas ${ }^{(21)}$.

Por último, se reconoce firmemente que, cuando se trata de trastornos de la deglución, los profesionales del lenguaje desempeñan un papel fundamental como miembros del equipo de tratamiento. Sin embargo, varios estudios e informes demuestran que esta área de la práctica está infrautilizada, poco reconocida y desarrollada. Se destaca la necesidad de investigación, for- mación y educación más centrada en el tema para estos profesionales $^{(18,22)}$.

\section{INDICACIONES NUTRICIONALES ESPECÍFICAS EN CP}

\section{Insuficiencia cardíaca (IC)}

La IC es un síndrome clínico, resultante de trastornos cardíacos que deterioran la función del miocardio. En la actualidad es un importante problema de salud pública, que amenaza a más de 20 millones de pacientes a nivel mundial ${ }^{(23)}$. La IC es la principal causa de morbilidad y mortalidad en todo el mundo, por lo que es fundamental que estos pacientes dispongan de una CP adecuada para mejorar su CdV, independientemente del pronóstico ${ }^{(24)}$. Los pacientes refieren con frecuencia disnea, moderada a grave; fatiga; tos; debilidad muscular; insomnio y bajo estado de ánimo. Asimismo, se observan limitaciones en las actividades de la vida diaria, que pueden derivar en una dependencia de los cuidados, a lo que se suman las múltiples comorbilidades asociadas y la polifarmacia ${ }^{(24)}$. A pesar de ello, las consultas de CP en estos pacientes siguen siendo insuficientes ${ }^{(25)}$.

El pronóstico de la IC es aún peor en los pacientes con desnutrición ${ }^{(26)}$. Estos suelen presentar una disminución del apetito ${ }^{(27,28)}$, lo que agrava la desnutrición asociada. La falta de apetito es variada; el proceso de envejecimiento puede llevar a una pérdida de hambre debido a la alteración de las hormonas del apetito y al deterioro del estado de salud, la depresión y los problemas gastrointestinales ${ }^{(26)}$.

\section{Enfermedad pulmonar obstructiva crónica (EPOC) avanzada}

La prevalencia de la EPOC, y la mayor supervivencia de estos pacientes, han aumentado el número de personas en estado terminal. Sin embargo, no se han desarrollado programas de $\mathrm{CP}$, lo que impide que los pacientes reciban una atención óptima y, por tanto, que se garantice su bienestar, lo que evita nuevos ingresos hospitalarios $^{(29)}$.

La desnutrición es un problema frecuente en los pacientes con EPOC. La prevalencia de desnutrición relacionada con la enfermedad se da en el 19,8\% de los pacientes con EPOC inicial. Esta condición aumenta en las fases más avanzadas de la enfermedad. Es importante destacar que la ingesta energética se ve dificultada por la falta de hambre en estos pacientes, unida a un aumento de la disnea al intentar comer. Además, suele 
haber una afectación de la percepción del gusto y problemas para masticar o tragar ${ }^{(30)}$.

Malo de Molina y colaboradores refieren en el Protocolo de tratamiento domiciliario del paciente con enfermedad pulmonar y EPOC avanzada, que debido a la alta prevalencia de bajo peso entre estos pacientes, su peso corporal debe ser monitorizado periódicamente, a través del indicador de índice de masa corporal ${ }^{(31)}$. Por otro lado, la prevalencia de sarcopenia en los pacientes con EPOC oscila entre el $14,5 \%$ y el $25 \%$, con variabilidad según la edad y la gravedad de la enfermedad. Se ha visto que en los pacientes con EPOC avanzada existe un cambio en la distribución de la fibra muscular tipo I a favor del tipo IIx en el cuádriceps y el tibial anterior. Este hecho no se observa habitualmente en el envejecimiento normal o en pacientes con EPOC leve ${ }^{(29)}$.

\section{Esclerosis lateral amiotrófica (ELA)}

La ELA es una enfermedad crónica, mortal y progresiva. El pronóstico depende del estado nutricional y respiratorio del paciente, por lo que el objetivo principal es maximizar su $\mathrm{CdV}^{(32)}$. Se ha descrito que una pérdida de peso del $5 \%$ en el momento del diagnóstico duplica el riesgo de muerte del paciente; asimismo, un aumento de más de 2,5 kg de masa grasa se asocia con una reducción del $10 \%$ del riesgo de muerte ${ }^{(32)}$. Son muchos los factores que influyen en la pérdida de peso corporal, entre ellos destacan la disfagia, el aumento del gasto energético (por el mayor trabajo respiratorio y el estado inflamatorio), la anorexia, la dificultad para manejar los utensilios necesarios para comer, el retraso en el vaciado gástrico, el estreñimiento, la depresión y la ansiedad. Es esencial destacar que la desnutrición por sí misma afecta negativamente a la fuerza muscular y a la inmunidad, lo que influye en la evolución de la enfermedad $^{(33)}$.

El manejo oportuno de la enfermedad es esencial para aliviar la desnutrición, la insuficiencia respiratoria y prevenir la aspiración, las caídas y la neumonía. Para controlar la pérdida de peso y satisfacer las necesidades nutricionales del paciente están indicados los suplementos nutricionales orales, cuando el paciente mantiene su capacidad de deglución. Además, se recomienda modificar la textura de los alimentos en un líquido espeso o pudín para mejorar la ingesta del paciente ${ }^{(33)}$. Es fundamental analizar la administración de la alimentación por sonda enteral de forma precoz, y considerar la colocación de una gastrostomía antes de que la respiración se vea comprometida o cuando los pacientes pierdan un $10 \%$ de su peso normal ${ }^{(34)}$. Es conveniente que la colocación de la PEG se realice antes de que la capacidad vital forzada (CVF) sea inferior al $50 \%$, aunque no haya problemas nutricionales ni evidencia de disfagia en ese momento. Asimismo, la gastrostomía radiológica percutánea puede realizarse con una CVF inferior al $50 \%$, ya que requiere un menor grado de sedación. Por otro lado, el uso de la NP se reserva para aquellos pacientes en los que la NE no es posible por su situación respiratoria de base ${ }^{(33)}$.

\section{Esclerosis múltiple (EM)}

La EM es una enfermedad progresiva y crónica, que afecta a 2,3 millones de pacientes en todo el mundo. Se trata de una enfermedad autoinmune e inflamatoria, con afectación de la vaina de mielina de las neuronas del cerebro y la médula espinal, con pérdida gradual de neuronas y axones ${ }^{(35)}$. Esta patología afecta al desempeño de las actividades cotidianas, a la autonomía del paciente y a su $\mathrm{CdV}^{(35)}$. Es común la presencia de pérdida de peso y condición de desnutrición en estadios avanzados de la EM, debido a complicaciones en la alimentación, pérdida de hambre, náuseas, aumento del gasto energético y proteico, incremento de las pérdidas relacionadas con la condición inflamatoria crónica, sarcopenia correlacionada con menor actividad física, afectación cognitiva, úlceras por presión, polifarmacia y disfagia neurógena. En consecuencia, la malnutrición empeora la fragilidad, lo que disminuye la capacidad funcional y produce más disfagia e infecciones, que conducen de nuevo a la malnutrición. Como se ha descrito anteriormente, la desnutrición incrementará la morbilidad y la mortalidad en estos pacientes ${ }^{(36)}$.

Aunque existe escasa información que apoye la incorporación de los CP como parte del protocolo de los pacientes con $\mathrm{EM}^{(35)}$, los factores mencionados anteriormente ponen de manifiesto las recomendaciones de la Sociedad Europea de Nutrición Clínica (ESPEN) para el asesoramiento nutricional, para prevenir y mejorar el estado nutricional en estos pacientes. El objetivo de ello será proporcionarles una ingesta adecuada de energía y proteínas, fibra, ácidos grasos poliinsaturados y vitamina $\mathrm{D}^{(36-39)}$.

\section{Enfermedad de Parkinson (EP)}

La EP es un trastorno neurodegenerativo, con una prevalencia mundial de casi el $1 \%$ de las personas a partir de la sexta década de vida. En estos pacientes, la desnu- 
trición se debe a múltiples factores, como la discinesia, la inelasticidad, la disfagia y los efectos secundarios de la medicación. La desnutrición y la neumonía por aspiración, causadas principalmente por la disfagia, se han descrito como una de las principales causas de muerte en los pacientes con $\mathrm{EP}^{(40)}$. La ESPEN recomienda la detección periódica de la disfagia en los pacientes con EP, incluso sin síntomas aparentes, debido a su importancia clínica.

La disfagia sarcopénica es frecuente en estos pacientes, y se produce por la disminución de la masa muscular y de la fuerza corporal total, lo que afecta al proceso de deglución. Por esta razón, la terapia combinada con la rehabilitación y el manejo nutricional es esencial. Esto enfatiza la relevancia de elaborar una intervención integral y multidisciplinar para tener resultados efectivos en estos pacientes, al aumentar el número de comidas al día, al adaptar los horarios de las comidas para los momentos con menos síntomas de EP, y al proporcionar comida casera (cuando sea posible) y tentempiés, para alcanzar las calorías necesarias diariamente ${ }^{(40)}$.

Dependiendo de la gravedad de la disfagia puede ser beneficioso adaptar las características de los bolos, las maniobras posturales, los programas de ejercicio ${ }^{(40)}$, entre otras intervenciones. Para aquellos pacientes con EP en estadios avanzados (estadios 4 y 5 de Hoehn y Yahr) se aconseja realizar un video fluoroscópico de la deglución, o una evaluación endoscópica con fibra óptica de la misma, para evaluar sus alteraciones e identificar la aspiración silenciosa. De este modo se puede adaptar la viscosidad de los alimentos y los líquidos. Cuando se incrementa la probabilidad de aspiración o atragantamiento con los alimentos se debe contemplar la nutrición junto con la SNG o la $\mathrm{PEG}^{(41)}$.

Es esencial vigilar los efectos secundarios de los medicamentos para la EP, ya que algunos de ellos producen vómitos, náuseas, dolor abdominal, estreñimiento, dispepsia, pérdida de peso, xerostomía, anorexia, diarrea y otras alteraciones gastrointestinales. Estas consecuencias indeseables también alteran el sabor y el olor de los alimentos, lo que contribuye al deterioro del estado nutricional ${ }^{(37)}$. Además, los pacientes con EP se benefician de la administración de suplementos de homocisteína, B12 y folato, ya que se ha informado de que la levodopa, uno de los medicamentos más comunes para la EP, afecta al metabolismo de los lípidos y los hidratos de carbono, y produce hiperhomocisteinemia, B12 y deficiencia de folato ${ }^{(37)}$. Además, se ha demostrado que los regímenes de repartición de proteínas promueven la eficacia de la levodopa, al afectar positivamente los síntomas motores y la puntuación de discapacidad, lo que mejora la $\mathrm{CdV}^{(42)}$.

Algunos informes ${ }^{(35,40,42,43)}$ proponen que los pacientes con EP tienen deficiencias que podrían abordarse con el siguiente enfoque: coenzima $Q_{10}(300-1200 \mathrm{ng}$ diarios), glutatión (dieta mediterránea, n-acetil cisteína $1200-8000 \mathrm{mg} /$ día), vitamina D (1200 UI diarias) y vitamina C (la dosis sugerida sigue sin estar clara).

La suplementación con 1 a $4 \mathrm{~g}$ de ácido graso omega 3 puede disminuir la inflamación y mejorar la $\mathrm{CdV}^{(44,45)}$. Dado que el estreñimiento es un síntoma frecuente, la dieta de los pacientes con EP debería incrementar el consumo de fibra y líquidos ${ }^{(42)}$. Los probióticos $8 \times 10^{9}$ UFC de bacterias vivas de varias especies pueden añadirse como medicamento complementario ${ }^{(43)}$.

\section{Pacientes con cáncer}

La prevalencia del cáncer está aumentando en todo el mundo, y es una de las principales causas de morbilidad y mortalidad. Asimismo, los diferentes tratamientos han permitido una mayor supervivencia en estos pacientes, convirtiéndose en una enfermedad crónica ${ }^{(46)}$. La desnutrición y la pérdida de masa muscular son frecuentes en los pacientes con cáncer, lo que influye en la evolución de la enfermedad. La ingesta nutricional inadecuada, la falta de actividad física y los trastornos metabólicos catabólicos empeoran la situación ${ }^{(47)}$.

La OMS exhorta a la integración temprana de las intervenciones en materia de $\mathrm{CP}$, desde el principio de la enfermedad hasta la muerte y más allá. El apoyo nutricional debe recibir una consideración especial si los pacientes están recibiendo tratamiento paliativo contra el cáncer ${ }^{(46)}$. Las directrices de la ESPEN recomiendan un tamizaje exhaustivo de todos los pacientes con cáncer avanzado, para detectar una ingesta nutricional inadecuada, pérdida de peso y un índice de masa corporal bajo ${ }^{(7,46)}$. Si el paciente ha sido identificado como de riesgo, o presenta desnutrición debido a una deficiencia, es necesario valorar el estado nutricional mediante la evaluación de la cantidad y la calidad de la ingesta nutricional ${ }^{(48)}$. Se deben analizar los signos y síntomas que puedan afectar negativamente el estado nutricional y el malestar psicosocial asociado con la alimentación o con la pérdida de peso. Druml y colaboradores refirieron que la nutrición en CP debe considerar aspectos sociales, culturales, emocionales y existenciales, como las necesidades espirituales y étnicas de los pacientes ${ }^{(7)}$. 
Los pacientes con cáncer pueden pertenecer a dos subpoblaciones muy diferentes: los pacientes que reciben terapia oncológica y los que tienen un cáncer "incurable", y no pueden recibir tratamientos oncológicos. Esta diferencia es esencial porque las posibles intervenciones nutricionales cambian según el resultado esperado.

\section{Cáncer y tratamiento oncológico}

En los pacientes que reciben quimioterapia, la mayoría de las veces se prescriben intervenciones nutricionales para mantener o mejorar el estado general, y disminuir la toxicidad de los medicamentos y la morbilidad en general $^{(49)}$. Los pacientes con cáncer gastrointestinal son un ejemplo muy claro de lo anterior, en el que la $\mathrm{NP}$, en casos de contraindicación del uso del tracto gastrointestinal o de NE, superando ciertos tipos de cáncer (esófago), puede permitir al paciente no solo recibir y completar tratamientos que, de otra manera, la inanición no permitiría o llevaría a la muerte antes de que lo haga la enfermedad primaria ${ }^{(10)}$.

Varias publicaciones destinadas a mostrar la mejora de la CdV en pacientes con intervenciones nutricionales, como la consejería nutricional, la suplementación $^{(50)}$, las fortificaciones y la NE, tuvieron resultados diferentes y contradictorios. Mientras que algunos de ellos muestran una mejora en los dominios de la CdV, otros no informaron de ningún efecto con las intervenciones. Los estudios sobre NP y, más concretamente, en la NP domiciliaria informaron de beneficios en los índices de $\mathrm{CdV}^{(6-9,46)}$.

\section{Cáncer como enfermedad terminal}

En estos pacientes, la hiporexia, la anorexia y la abstinencia de ingerir alimentos y líquidos forman parte del proceso de muerte. La mayoría de estos pacientes no experimentan hambre ni sed, como temen sus familiares y cuidadores ${ }^{(46)}$. La evidencia no justifica la terapia nutricional, sobre todo si se tiene que administrar como NHA. Se cree que las vías enteral o parenteral aumentan el sufrimiento y las complicaciones, ya que la terapia estándar no mejora ni cambia los resultados de la enfermedad ${ }^{(46)}$.

Sin embargo, algunos autores han demostrado que, en particular en los pacientes con cáncer gastrointestinal incurable y afectación peritoneal que provoca una obstrucción intestinal crónica, la NP a domicilio puede permitir que los pacientes abandonen las instalaciones hospitalarias y sobrevivan sin síntomas en una pobla- ción en la que la inanición, si no se trata, puede causar la muerte antes que el propio tumor ${ }^{(10)}$. Como ya se mencionó, en estos casos, las directrices de la ESPEN recomiendan intervenir individualmente, respetando los aspectos individuales, culturales o religiosos ${ }^{(7)}$.

\section{Pacientes de edad avanzada}

La población anciana tiene mayor riesgo de desnutrición y deshidratación. Además, el envejecimiento incluye el avance de muchas enfermedades crónicas, multimorbilidad, polimorfismo y discapacidades psicosociales. En esta población es muy común encontrar no solo dificultades para ingerir alimentos, sino falta de diversidad en los nutrientes ingeridos. No comer o beber lo necesario se debe tomar en cuenta como una decisión autónoma que expresa una fase de muerte ${ }^{(51)}$.

Todo lo anterior plantea una decisión ética muy compleja sobre qué opciones médicas o terapéuticas son más adecuadas. En sus directrices, la ESPEN recomienda algunos principios que deben guiar estas decisiones. Uno de ellos es el principio de autonomía, el hecho de que la ingesta de alimentos debe significar un aspecto positivo de la vida de las personas mayores, de lo contrario, es inútil. También es importante distinguir entre la falta de apetito y la depresión, u otros problemas psicosociales que pueden tratarse ${ }^{(7)}$.

Puede ser un reto tener una visión clara de las alternativas la mayoría de las veces. Como ya se ha dicho, una buena comunicación entre todos los actores implicados, y el respeto de la autonomía de los pacientes, es fundamental para un cuidado exitoso. Mientras tanto, en aquellos casos en los que las decisiones bien fundamentadas son difíciles, la preservación de la vida es la $\operatorname{prioridad}^{(7)}$.

\section{Pacientes con demencia}

La demencia es un trastorno progresivo, limitante de la vida y sin terapéutica curativa, que se caracteriza por un deterioro, que perturba las actividades de la vida diaria. Puede afectar a la memoria, el lenguaje, la función ejecutiva, la planificación de tareas y la ejecución de los pacientes ${ }^{(52)}$, e incluye más de 200 trastornos neurológicos graduales de gravedad variable. En función de ello, el paciente necesitará más ayuda para atender sus necesidades. Los estadios avanzados de la enfermedad se distinguen por un profundo deterioro cognitivo, incapacidad para interactuar verbalmente y dependencia funcional total ${ }^{(14)}$. 
A estos pacientes no se les suele reconocer un alto riesgo de muerte, por lo que los $\mathrm{CP}$ no siempre se consideran para su tratamiento estándar. Como se mencionó, con la progresión de la enfermedad, los pacientes requerirán cada vez más asistencia para sus necesidades diarias. En cuanto a los requerimientos nutricionales, uno de los problemas más frecuentes en ellos es la desnutrición, a pesar del estadio de la enfermedad ${ }^{(14,53)}$. Es fundamental proporcionar una nutrición adecuada, aportando las calorías necesarias para evitar la pérdida de peso. Se pueden implementar varias acciones para alcanzar ese objetivo: orientación por parte de los nutricionistas a los cuidadores sobre la preparación de una dieta acorde a las necesidades del paciente, evaluación nutricional constante, suplementación nutricional oral, cuando sea necesario, y tras un cuidadoso consenso, $\mathrm{NE}$ o NP ${ }^{(53)}$.

En estadios más avanzados, los ingresos hospitalarios no programados, los trastornos alimentarios con rechazo a comer, el déficit motor, la pérdida de peso o la disfagia severa pueden ser indicadores de demencia avanzada, por tanto, se debe considerar la posibilidad de una alimentación invasiva para satisfacer los requerimientos nutricionales ${ }^{(14,53)}$. Sin embargo, debe ponderarse el mayor riesgo concomitante de infección del sitio de la PEG y de aspiración para planificar un tratamiento individualizado para estos pacientes, contemplando las cuestiones culturales y los deseos de los pacientes.

Los estudios científicos no han podido demostrar que la alimentación e hidratación artificial tengan algún beneficio para prolongar la vida, modificar positivamente la CdV o prevenir el sufrimiento. Además, no se han encontrado menores tasas de aspiración. Por el contrario, se ha demostrado que sus cargas superan a los beneficios debido a la naturaleza invasiva de los procedimientos y a la necesidad de sujeciones, lo que paradójicamente aumenta el sufrimiento ${ }^{(54,55)}$.

En este caso, los pacientes con demencia y dificultades para comer necesitan apoyo, de un plan individualizado y de una asistencia alimentaria cuidadosa para evitar, en la medida de lo posible, el riesgo de aspiración. Para ayudar a la familia y al paciente a tomar una mejor decisión es fundamental proporcionar información sobre las opciones de alimentación, la nutrición de confort cerca del FdV, el riesgo y los beneficios de las sondas de alimentación y la alimentación oral asistida ${ }^{(14)}$. Al igual que con las demás enfermedades, la comunicación y las palabras deben evitar las connotaciones negativas; por ello se puede utilizar "alimentación de confort" para referirse a este tipo de terapia nutricional ${ }^{(7)}$.

\section{Estado vegetativo persistente}

El diagnóstico, el pronóstico y la posibilidad de recuperación, así como el alcance de los cuidados que debe recibir este paciente, hacen que estos casos se deban evaluar individualmente. Si el pronóstico es incierto, se recomienda que la NHA forme parte del tratamiento. Como siempre, hay que comunicar cuidadosamente los beneficios del tratamiento y los objetivos a alcanzar. Si existe o se presume una voluntad expresa, esta debe respetarse ${ }^{(7)}$.

\section{Enfermedad renal crónica (ERC)}

La ERC es un importante problema de salud pública, que está creciendo en todo el mundo ${ }^{(12)}$. Estos pacientes suelen tener múltiples comorbilidades asociadas, que deben ser abordadas en profundidad.

El enfoque nutricional del paciente con ERC suele basarse en la prolongación de la vida con dietas muy controladas, que incluyen la restricción de la ingesta de electrlitos, líquidos y macronutrientes. Sin embargo, el manejo dietético en los CP debe estar alineado con los objetivos del paciente y su CdV, siendo frecuente el manejo prioritario de los síntomas presentes. Puede ser aconsejable, si es necesario, el uso de suplementos nutricionales orales.

La sarcopenia es frecuente en estos pacientes, lo que conlleva fragilidad y pérdida de independencia. Es fundamental incorporar programas de ejercicio estructurados que incorporen entrenamiento de resistencia y mejoren el estado cardiorrespiratorio, la fuerza muscular y la capacidad funcional, junto con una ingesta nutricional adecuada ${ }^{(12)}$. La anorexia es frecuente en los pacientes con ERC, y conduce a la desnutrición, lo que aumenta la mortalidad. La prevención y el tratamiento de la desnutrición deben centrarse en estrategias que reduzcan los síntomas, optimicen la ingesta dietética, promuevan una función intestinal saludable y apoyen la función física y la independencia ${ }^{(56)}$. Es necesario abordar los síntomas urémicos, como la xerostomía, las alteraciones del gusto, las náuseas, la sequedad y los vómitos, y los trastornos de la motilidad, que pueden conducir a una menor ingesta de alimentos ${ }^{(12)}$.

En el caso de los pacientes que se encuentran en la fase de FdV es esencial evitar ser excesivamente prescriptivo o restrictivo, y proporcionar consejos prácticos, que tengan en cuenta los objetivos individuales y las necesidades psicosociales y culturales ${ }^{(12)}$. Durante esta etapa, el plan nutricional debe estar centrado en el paciente, para maximizar el placer de la comida y redu- 
cir las molestias relacionadas con la misma ${ }^{(8)}$. No hay pruebas sólidas que respalden la recomendación de NE para los pacientes al FdV, ya que no reduce la carga de síntomas ni promueve la $\mathrm{CdV}^{(57)}$.

\section{CONCLUSIONES}

Se han descrito las recomendaciones nutricionales generales en los CP, sus complicaciones y su impacto en la CdV. Además, se ha presentado la percepción de los familiares y cuidadores sobre las mismas y sus aspectos éticos, resaltando la importancia de que las recomendaciones nutricionales en los CP estén centradas en el paciente y en la comunicación abierta entre las partes interesadas.

El cuidado nutricional en los CP puede incluir intervenciones tempranas para reducir los síntomas del estado nutricional, implementar el asesoramiento nutricional, el uso de suplementos nutricionales orales y la NHA. El cuidado nutricional (oral, NE, NP o intervenciones combinadas) debe prescribirse de acuerdo con el estadio de la enfermedad del paciente, los requerimientos energéticos y proteicos, considerando la tolerabilidad y, sobre todo, respetando los deseos del paciente, familia o cuidador.

Es fundamental destacar que un equipo multidisciplinar debe proponer un tratamiento nutricional individualizado, previa explicación exhaustiva de las ventajas e inconvenientes de las opciones de alimentación al paciente, familiar o cuidador, para que este dé su consentimiento informado. La NE y NP se deben evaluar cuidadosamente para la condición de cada paciente, especialmente aquellos en situación de FdV, ya que la intención principal en ellos es la CdV, más que lograr los objetivos nutricionales.

Se recomienda a los pacientes con condiciones específicas, que impliquen deterioro cognitivo, planificar con antelación sus preferencias de alimentación e intervenciones en situaciones futuras, que se presentarán a medida que la enfermedad siga su curso natural.

\section{Conflictos de intereses}

Los autores certifican que no existe ningún conflicto de intereses con ninguna organización financiera en relación con el material tratado en el manuscrito.

\section{Financiación}

Esta investigación no ha recibido ninguna subvención específica de ningún organismo de financiación, ni del sector comercial ni del sector sin ánimo de lucro.

\section{Contribuciones de los autores}

Las responsabilidades de los autores fueron las siguientes: GM y LB fueron responsables del concepto de este trabajo y redactaron el manuscrito; GM, LB, MC, FC proporcionaron una revisión crítica del trabajo. Todos los autores contribuyeron y estuvieron de acuerdo con la versión final del manuscrito.

\section{Referencias}

1. Schwartz DB, Armanios N, Monturo C, Frankel EH, Wesley JR, Patel M, et al. Clinical ethics and nutrition support practice: Implications for practice change and curriculum development. J Acad Nutr Diet. 2016;116(11):1738-46. doi: 10.1016/j.jand.2016.01.009.

2. Schwartz DB, Olfson K, Goldman B, Barrocas A, Wesley JR. Incorporating palliative care concepts into nutrition practice: Across the age spectrum. Nutr Clin Pract. 2016;31(3):30515. doi: $10.1177 / 0884533615621556$.

3. Palliative care [Internet]. World Health Organization. [Consultado el 09 de abril de 2016]. Disponible en: http:// www.who.int/cancer/palliative/definition/en/

4. Boyce B. An Ethical perspective on palliative care. J Acad Nutr Diet. 2017 ;117(6):970-2. doi: 10.1016/j.jand.2017.01.017.

5. Van de Vathorst S. Artificial nutrition at the end of life: Ethical issues. Best Pract Res Clin Gastroenterol. 2014;28(2):247-53. doi: 10.1016/j.bpg.2014.02.005.

6. Schütte K, Middelberg-Bisping K, Schulz C. Nutrition and gastroenterological support in end of life care. Best Pract Res Clin Gastroenterol. 2020;48-49:101692. doi: 10.1016/j. bpg.2020.101692.

7. Druml C, Ballmer PE, Druml W, Oehmichen F, Shenkin A, Singer $\mathrm{P}$, et al. ESPEN guideline on ethical aspects of artificial nutrition and hydration. Clin Nutr. 2016;35(3):545-56. doi: 10.1016/j.clnu.2016.02.006.

8. McGinley E. Role of nutrition in the final stages of palliative care. J Community Nurs. 2015;29(1):53.

9. Ruggeri E, Giannantonio M, Agostini F, Ostan R, Pironi L, Pannuti R. Home artificial nutrition in palliative care cancer patients: Impact on survival and performance status. Clin Nutr. 2020;39(11):3346-53. doi: 10.1016/j.clnu.2020.02.021.

10. Bozzetti F. Is there a place for nutrition in palliative care? Support Care Cancer. 2020;28(9):4069-75. doi: 10.1007/ s00520-020-05505-x.

11. Hobson EV, McDermott CJ. Supportive and symptomatic management of amyotrophic lateral sclerosis. Nat Rev Neurol. 2016;12(9):526-38. doi: 10.1038/nrneurol.2016.111.

12. Stevenson J, Meade A, Randall AM, Manley K, Notaras S, Heaney $S$, et al. Nutrition in renal supportive care: Patientdriven and flexible. Nephrology. 2017;22(10):739-47. doi: 10.1111/nep.13090. 
13. Cotogni P, Caccialanza R, Pedrazzoli P, Bozzetti F, De Francesco A. Monitoring response to home parenteral nutrition in adult cancer patients. Healthcare. 2020;8(2):183. doi: $10.3390 /$ healthcare 8020183 .

14. Murphy E, Froggatt K, Connolly S, O’Shea E, Sampson EL, Casey D, et al. Palliative care interventions in advanced dementia. Cochrane Database Syst Rev. 2016;12(12):CD011513. doi: 10.1002/14651858.CD011513.pub2.

15. Yamagishi A, Morita T, Miyashita M, Sato K, Tsuneto S, Shima $\mathrm{Y}$. The care strategy for families of terminally ill cancer patients who become unable to take nourishment orally: recommendations from a nationwide survey of bereaved family members' experiences. J Pain Symptom Manage. 2010;40(5):67183. doi: 10.1016/j.jpainsymman.2010.02.025.

16. François K, Lobb E, Barclay S, Forbat L. The nature of conflict in palliative care: A qualitative exploration of the experiences of staff and family members. Patient Educ Couns. 2017;100(8):1459-65. doi: 10.1016/j.pec.2017.02.019.

17. Amano K, Baracos VE, Hopkinson JB. Integration of palliative, supportive, and nutritional care to alleviate eating-related distress among advanced cancer patients with cachexia and their family members. Crit Rev Oncol Hematol. 2019;143:117-23. doi: 10.1016/j.critrevonc.2019.08.006.

18. Moloney J, Walshe M. Managing and supporting quality-oflife issues in dysphagia: A survey of clinical practice patterns and perspectives in the UK, Ireland and South Africa. Int J Lang Commun Disord. 2019;54(1):41-9. doi: 10.1111/14606984.12429.

19. Langmore SE, Grillone G, Elackattu A, Walsh M. Disorders of swallowing: Palliative care. Otolaryngol Clin North Am. 2009;42(1):87-105. doi: 10.1016/j.otc.2008.09.005.

20. Patterson JM, Rapley T, Carding PN, Wilson JA, McColl E. Head and neck cancer and dysphagia; caring for careers. Psychooncology. 2013;22(8):1815-20. doi: 10.1002/ pon.3226.

21. Smith BJ, Chong L, Nam S, Seto R. Dysphagia in a palliative care setting-a coordinated overview of caregivers' responses to dietary changes: The DysCORD qualitative study. J Palliat Care. 2015;31(4):221-7. doi: 10.1177/082585971503100403.

22. O'Reilly AC, Walshe M. Perspectives on the role of the speech and language therapist in palliative care: An international survey. Palliat Med. 2015;29(8):756-61. doi: $10.1177 / 0269216315575678$.

23. Yu DSF. Effective management for older people with heart failure: from acute to palliative care paradigms. J Geriatr Cardiol. 2016;13(5):391-2. doi: 10.11909/j.issn.16715411.2016.05.007.

24. Janssen DJA, Johnson MJ, Spruit MA. Palliative care needs assessment in chronic heart failure. Curr Opin Support Palliat Care. 2018;12(1):25-31. doi: 10.1097/ SPC. 0000000000000317.

25. Von Schwarz ER, He M, Bharadwaj P. Palliative care issues for patients with heart failure. JAMA Netw Open. 2020;3(2):e200011. doi: 10.1001/jamanetworkopen.2020.0011.

26. Andreae C, Strömberg A, Årestedt K. Prevalence and associated factors for decreased appetite among patients with stable heart failure. J Clin Nurs. 2016;25(11-12):1703-12. doi: 10.1111/jocn.13220.

27. Chan HYL, Yu DSF, Leung DYP, Chan AWK, Hui E. Quality of life and palliative care needs of elderly patients with advanced heart failure. J Geriatr Cardiol. 2016;13(5):420-4. doi: 10.11909/j.issn.1671-5411.2016.05.016.

28. Diop MS, Rudolph JL, Zimmerman KM, Richter MA, Skarf LM. Palliative care interventions for patients with heart failure: A systematic review and meta-analysis. J Palliat Med. 2017;20(1):84-92. doi: 10.1089/jpm.2016.0330.

29. Pérez de Llano LA. Cuidados paliativos para pacientes con insuficiencia respiratoria crónica. Gac Sanit. 2018;32(4):3835. doi: http://dx.doi.org/10.1016/j.gaceta.2018.04.006.

30. Vázquez-Espinosa E, López RC, Sampedro-Núñez M. Alteración nutricional del paciente con EPOC. Etiopatogenia. Consecuencias de la desnutrición en el paciente con EPOC. Rev Patol Respir. 2018;21(2):S166-S17.

31. Malo de Molina RR, Valle FM, Ussetti GP. Protocolo de tratamiento domiciliario del paciente con enfermedad pulmonar obstructiva crónica avanzada. Medicine. 2014;11(65):3894900. doi: 10.1016/S0304-5412(14)70859-5.

32. Danel-Brunaud V, Touzet L, Chevalier L, Moreau C, Devos D, Vandoolaeghe S, et al. Ethical considerations and palliative care in patients with amyotrophic lateral sclerosis: A review. Rev Neurol. 2017;173(5):300-7. doi: 10.1016/j.neurol.2017.03.032.

33. Del Olmo GMD, Virgili CN, Cantón BA, Lozano FFM, Wanden-Berghe C, Avilés V, et al. Manejo nutricional de la esclerosis lateral amiotrófica: resumen de recomendaciones. Nutr Hosp. 2018;35(5):1243-51. doi: 10.20960/nh.2162.

34. Karam CY, Paganoni S, Joyce N, Carter GT, Bedlack R. Palliative care issues in amyotrophic lateral sclerosis: An evidenced-based review. Am J Hosp Palliat Care. 2016;33(1):8492. doi: $10.1177 / 1049909114548719$.

35. Latorraca OC, Martimbianco CAL, Pachito DV, Torloni MR, Pacheco RL, Pereira GJ, et al. Palliative care interventions for people with multiple sclerosis. Cochrane Database Syst Rev. 2019;10(10):CD012936. doi: 10.1002/14651858. CD012936.pub2.

36. Redondo RL, Pintor de la MB, Tejada GJ, García VJJ, Fernández GMJ, Barrera MI, et al. Nutritional profile of multiple sclerosis. Nutr Hosp. 2019;36(2):340-9. doi: 10.20960/ nh.2023.

37. Burgos R, Bretón I, Cereda E, Desport JC, Dziewas R, Genton $\mathrm{L}$, et al. ESPEN guideline clinical nutrition in neurology. Clin Nutr. 2018;37(1):354-96. doi: 10.1016/j.clnu.2017.09.003.

38. Riccio P, Rossano R. Diet, gut microbiota, and vitamins D + A in multiple sclerosis. Neurotherapeutics. 2018;15(1):75-91. doi: 10.1007/s13311-017-0581-4. 
39. Di Somma C, Scarano E, Barrea L, Zhukouskaya VV, Savastano S, Mele C, et al. Vitamin D and neurological diseases: An endocrine view. Int J Mol Sci. 2017;18(11):2482. doi: $10.3390 /$ ijms 18112482 .

40. Yamada Y, Shamoto H, Maeda K, Wakabayashi H. Homebased combined therapy with rehabilitation and aggressive nutrition management for a Parkinson's disease patient with sarcopenic dysphagia: A case report. Prog Rehabil Med. 2018;3:20180019. doi: 10.2490/prm.20180019.

41. Umemoto G, Furuya H. Management of dysphagia in patients with Parkinson's disease and related disorders. Intern Med. 2020;59(1):7-14. doi: 10.2169/internalmedicine.2373-18.

42. Gatti S, Carugi M, Palermo V, Casellato C, Gambini C, Zuin $M$, et al. Nutritional assessment in patients with Parkinson's disease: The nutri-park study. Nutr Health Aging. 2020;5(4):297-305. doi: 10.3233/NHA-200083.

43. Lister $\mathrm{T}$. Nutrition and lifestyle interventions for managing Parkinson's disease: A narrative review. J Mov Disord. 2020;13(2):97-104. doi: 10.14802/jmd.20006.

44. Muscogiuri G, Altieri B, Annweiler C, Balercia G, Pal HB, Boucher BJ, et al. Vitamin D and chronic diseases: the current state of the art. Arch Toxicol. 2017;91(1):97-107. doi: 10.1007/s00204-016-1804-x.

45. Tamtaji RO, Taghizadeh M, Aghadavod E, Mafi A, Dadgostar E, Daneshvar KR, et al. The effects of omega-3 fatty acids and vitamin $\mathrm{E}$ co-supplementation on gene expression related to inflammation, insulin and lipid in patients with Parkinson's disease: A randomized, double-blind, placebo-controlled trial. Clin Neurol Neurosurg. 2019;176:116-21. doi: 10.1016/j.clineuro.2018.12.006.

46. Arends J, Bachmann P, Baracos V, Barthelemy N, Bertz H, Bozzetti $\mathrm{F}$, et al. ESPEN guidelines on nutrition in cancer patients. Clin Nutr. 2017;36(1):11-48. doi: 10.1016/j. clnu.2016.07.015.

47. Rodríguez VD, Frías-Toral E, Santana PS. Requerimientos ajustados a la desnutrición del paciente oncológico. Rev Oncol Ecu. 2019;29(2):83-96. doi: https://doi.org/10.33821/92.

48. Rodríguez VD, Frías-Toral E, Santana PS. Diagnóstico nutricional en el paciente oncológico: importancia y herramien- tas para realizarla. Rev Oncol Ecu. 2018;28(3):169-80. doi: https://doi.org/10.33821/271.

49. Moreno F, García C, Veintimilla D, Frías E, Moreno A. Valoración nutricional de pacientes oncológicos que inician tratamiento quimioterápico. Rev Oncol Ecu. 2011;21(34):15-22.

50. Rodríguez VD, Guerrero TM, Maldonado VM, Herrera S, Frías-Toral E. Importancia del uso de suplementos nutricionales orales en pacientes con cáncer colorrectal. Reporte de caso. Rev Oncol Ecu. 2015;25(1):51-4.

51. Volkert D, Beck AM, Cederholm T, Cruz-Jentoft A, Goisser $S$, Hooper L, et al. ESPEN guideline on clinical nutrition and hydration in geriatrics. Clin Nutr. 2019;38(1):10-47. doi: 10.1016/j.clnu.2018.05.024.

52. Connor J. Diagnosis and management of dementia in older people. Medicine. 2021;49(1):22-5. doi: 10.1016/j. mpmed.2020.10.005.

53. García-Soldevilla MA, Barragán MD, Rojo SA, Ayuso PL, Tejeiro MJ. Decisiones terapéuticas complejas en el anciano con demencia. Medicine. 2019;12(74):4381-4. doi: 10.1016/j.med.2019.03.018.

54. American Geriatrics Society Ethics Committee and Clinical Practice and Models of Care Committee. American Geriatrics Society feeding tubes in advanced dementia position statement. J Am Geriatr Soc. 2014;62(8):1590-3. doi: 10.1111/ jgs.12924.

55. Sampson EL, Candy B, Jones L. Enteral tube feeding for older people with advanced dementia. Cochrane Database Syst Rev. 2009;2009(2):CD007209. doi: 10.1002/14651858. CD007209.pub2.

56. Davison SN, Jassal SV. Supportive care: Integration of patientcentered kidney care to manage symptoms and geriatric syndromes. Clin J Am Soc Nephrol. 2016;11(10):1882-91. doi: 10.2215/CJN.01050116.

57. Good P, Richard R, Syrmis W, Jenkins-Marsh S, Stephens J. Medically assisted nutrition for adult palliative care patients. Cochrane Database Sys Revs. 2014;2014(4):CD006274. doi: 10.1002/14651858.CD006274.pub3. 